\title{
On moments and tail behaviors of storage processes
}

\author{
Arturo K ohatsu-Higa" \\ Universitat Pompeu Fabra, Department of E conomics \\ and B usiness, Ramón Trias Fargas 25-27, 08005 Barcelona, Spain \\ Makoto Yamazato \\ University of the Ryukyus, Department of $M$ athematics, Faculty of Science, \\ Senbaru1, Nishihara-cho, O kinawa, J apan 903-0213.
}

A pril 6, 2003

${ }^{x}$ The author was partially supported by grants BFM 2000-807 and BF M 2000-0598. 


\begin{abstract}
A bstract
We study the existence of moments and thetail behaviour of the densities of storage processes. We give suф cient conditions for existence and non-existence of moments using the integrability conditions of submultiplicative functions with respect to Lévy measures. Then, we study the asymptotical behavior of the tails of these processes using the concave or convex envelope of the release rate function.
\end{abstract}

ity.

K eywords: dam process, storage process, subordinator, submultiplicative functions, subexponentialJEL: E22 


\section{Introduction}

Storage processes are stochastic processes $f X(t) g$ de..ned through a stochastic dixerential equation of the type

$$
X(t)=x i^{Z_{t}} r(X(s)) d s+A(t):
$$

Here, $A$ is an increasing stochastic process called in put process and $r$ is a non-negative function, usually called the release rate. The solution of this type of stochastic dixerential equation has applications in storage systems theory, economics and insurance risk theory. For example, A can represent the amount of items arriving at a storage, the amount of rain that a dam receives or the amount of stochastic interest accrued by an account. The function $r$ can represent the way and/or rate the stored items are sold or delivered, how the water is released or the amount of money that is being used. Some references to possible applications can be found as early as K endall (1957) and in Desmukh and Pliska (1980).

In this article we are interested in a mathematical property related to storage processes with increasing Lévy processes as inputs. That is, ..rst we study the existence of moments and second, we study asymptotics of tail probabilities of $X$.

We will study the nonexistence (in Section 3) and the existence (in Section 4) of $E(g(X(t)))$ for a positive function $\mathrm{g}$. In various cases $\mathrm{g}$ will be a submultiplicative function. Power functions are particular cases of submultiplicative functions. In fact, our results applied to these particular examples give the

\begin{tabular}{|c|c|c|}
\hline$r(y)=y^{\circledR}$ & $g(y)=y$ & Criteria \\
\hline $0 \cdot \AA \cdot \quad 1$ & $>0$ & $y^{-0} \cong(d y)$ \\
\hline (R) $>1$ & 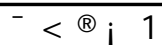 & always ..nite \\
\hline$\AA>1$ & $=\AA_{\mathrm{i}} 1$ & ${ }_{1}^{+1} \log y^{0}(d y)$ \\
\hline (B) $>1$ & ${ }^{-}>\mathbb{R}_{\mathrm{i}} 1$ & ${ }^{+1} \mathrm{y}^{-} \mathrm{i}^{\circledR+1} \mathrm{o}(\mathrm{dy})$ \\
\hline
\end{tabular}
following table:

The above criteria determines which moments of $X(t)$ are ..nite or in..nite.

Although these results obviously give some information on the tail probabilities of these processes, we give more precise asymptotics (exact order) of tail probabilities of storage processes via their Lévy measures in Section 6. To do this, the concept subexponentiality ([3]) plays an important role. Our results show that process of Ornstein-Uhlenbeck type $(r(y)=$ ay case) occupies a critical position on the tail behavior (Theorem 6.2). A result similar in part to one of our results (Theorem 6.3) has been obtained by Grigoriu and Samorodnitsky [8] under a dixerent assumption.

A rticles related with the properties we study here are Asmussen (1998) where the tail behaviour of the stationary distribution of storage processes and the distribution of the ruin time of risk processes is investigated. In Sigman and Yao (1994) the existence of moments for storage processes is studied although this study does not cover stable processes while ours does.

Possible applications of these results are in statistical properties of parameter estimators, simulation and numerical analysis of these systems such as weak or strong approximation results.

\section{Preliminaries}

In this section we describe how to construct a solution to (1). For further details, we refer to [2]. We assume the following hypotheses for $r$ throughout the article.

$(H 0) r:[0 ; 1) ![0 ; 1)$ such that $r(0)=0, r(x)>0$, for $x>0$, left continuous and $\lim _{y !} x+r(y)>0$ for all $x>0$.

We call $r(x)$ a release rate. $L$ et $f A(t) g$ be an increasing cadlag Lévy process such that $A(0)=0$ and

$$
E\left[e^{i \mu A(t)}\right]=\operatorname{expf}_{0}^{Z_{1}} t\left(e^{i \mu y} i 1\right)^{\circ}(d y) g ; \mu, 0
$$


where 0 is a measure on $(0 ; 1)$ satisfying

$$
0<Z_{0}^{Z_{1}}\left(x^{\wedge} 1\right) \circ(d x)<1:
$$

This measure 0 is called the Lévy measure of $f A(t) g$.

The idea of the construction is to ..rst treat the case of ...nite number of jumps. This can be solved explicitly pathwise. Finally one takes limits in the number of jumps in order to de.ne a solution to (1).

Consider ..rst the simple case of,$=0(0 ;+1)<+1$. Then the number of jumps of $A$ is ..nite in any compact interval. Denote the jump times and the jump sizes by $T_{n}$ and $Y_{n}, n=1 ; \cdots ;$, respectively. The interarrival times are denoted by $i_{n}=T_{n} i T_{n_{i}}$. In between two jumps $X$ is a solution of an ordinary dixerential equation that can be written using some auxiliary function q which we de.ne now. Set

$$
R(x ; y)={ }_{x}^{Z_{y}} \frac{1}{r(z)} d z
$$

for $0<x \cdot y$. De..ne $R(0 ; y):=R(0+; y) \cdot 1$. Since the function $R(x ; y)$; for $0<x \cdot y$; is continuous and strictly decreasing in $x$, it has a cont inuous inverse $R_{y}^{i}{ }^{1}(t)$ for $t 2[0 ; R(0 ; y))$. De.ne $q(t ; y)$ by

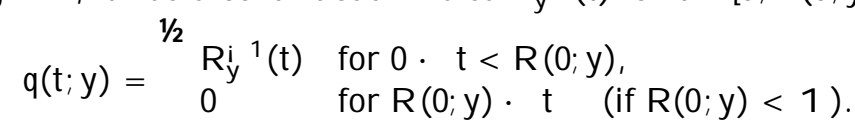

Then, q satis..es the following properties:

1. $q(R(x ; y) ; y)=x$ for $0<x \cdot y<1$ and $R(q(t ; y) ; y)=t$ for $y>0$ and $0 \cdot t<R(0 ; y)$.

2. $q(t ; y)$ is continuous, decreasing in $t$ and increasing in $y$.

3. Since $R(x ; y)$ is nondecreasing and left dixerentiable in $x, q(t ; y)$ is right dixerentiable in $t$ and it satis..es

$$
\begin{aligned}
\frac{d^{+}}{d t} q(t ; y) & =i r(q(t ; y)) ; \\
q(0 ; y) & =y ;
\end{aligned}
$$

for $x>0$ and $0 \cdot t<R(x ; 0)$.

Under this situation the solution $X$ of (1) is given by

$$
\begin{aligned}
X(0) & =X \\
X\left(T_{m}\right) & =q\left(i_{m} ; X\left(T_{m_{i} 1}\right)\right)+Y_{m} \quad(m, 1) ; \\
X(t) & =q\left(t ; T_{m} ; X\left(T_{m}\right)\right) \text { for } T_{m}<t<T_{m+1}:
\end{aligned}
$$

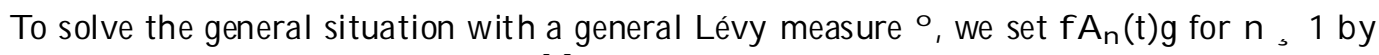

$$
A_{n}(t)={ }_{s \cdot t}^{X}\left(A(s) \text { i } A\left(s_{i}\right)\right) 1_{f A(s) i} A\left(s_{i}\right)>\frac{1}{n} g:
$$

Then, $f A_{n}(t) g$ is an increasing Lévy process with Lévy measure $o_{n}(\phi)=o\left(\phi \backslash\left(\frac{1}{n} ; 1\right)\right)$ with, $n=$ $o_{n}(0 ; 1)<+1$. For each $n, 1$, there is unique process $f X_{n}(t) g$ satisfying $(1)$. Since $A_{n}(t)$ is nondecreasing in $n, f X_{n}(t) g$ is also non decreasing in $n$. One can therefore de..ne $X(t)=\lim _{n} ! 1 X_{n}(t)$. Then, $f X(t) g$ satis..es (1) with driving noise $f A(t) g$. This process $f X(t) g$ is called a storage process starting at $x$ corresponding to $r$ and 0 . This process is a Hunt process. We call the L évy process $f A(t) g$ input process of $f X(t) g$. We denote $X(t)$ starting at $x$ by $X(t ; X)$ if necessary. For uniqueness and any further details we refer the reader to [2].

We denote the distribution of the i.i.d. random variables $Y_{n}$ by $F_{n}=, i \underline{1}_{n}$. The random variables $i_{k}=T_{k} i T_{k_{i} 1}, k, 1$ are i.i.d. with identical density, $n$ ei,nt. The sequences $f Y_{k} g$ and $f_{i k} g$ are mutually ind ependent. 


\section{Non-existence of moments}

In this and the next sections, we study relations between the tail behavior of the Lévy measure and the $R_{1}^{x i s t e n c e ~ o f ~ t h e ~ m o m e n t s ~ o f ~} f X(t) g$. We show that there is a remarkable dixerence between the two cases $R_{1} \frac{1}{r(y)} d y<1$ and ${ }_{1} \frac{1}{r(y)} d y=1$ (Examples $3.1 \gg 4.2$. See also the table in the Introduction).

Lemma 3.1 Let $\mathrm{g}$ be a nonnegative and nondecreasing function on $[0 ; 1)$. Let, $=\stackrel{o}{o}((1 ; 1))$. Then

$$
E[g(X(t ; x))], g(q(t ; x)) e^{i}, t+{ }_{1}^{Z_{1}} Z_{0} e^{e} \cdot s g(q(s ; y)) d s g=(d y)
$$

for all $x ; y, 0$.

Proof Let $f X_{1}(t) g$ be a storage process corresponding to $f A_{1}(t) g$ de. ned in Section 2. Then $X(t ; x)$, $\mathrm{X}_{1}(\mathrm{t} ; \mathrm{x})$ and we have

$$
\begin{aligned}
E[g(X(t ; x))], & E\left[g\left(X_{1}(t ; x)\right)\right] \\
= & X_{n=0}^{1} E\left[g\left(X_{1}(t ; x)\right): T_{n} \cdot t<T_{n+1}\right]:
\end{aligned}
$$

Since

$$
X_{1}(t ; x), q\left(t ; T_{n} ; q\left(T_{n} ; x\right)+Y_{n}\right) \text { on } f T_{n} \cdot t<T_{n+1} g \text {, }
$$

we have

$$
E\left[g\left(X_{1}(t ; x)\right): t<T_{1}\right]=g(q(t ; x)) e^{j \cdot t}
$$

and

$$
\begin{aligned}
& E\left[g\left(X_{1}(t ; x)\right): T_{n} \cdot t<T_{n+1}\right] \\
& E\left[g\left(q\left(t ; T_{n} ; q\left(T_{n} ; x\right)+Y_{n}\right)\right): T_{n} \cdot t<T_{n+1}\right]
\end{aligned}
$$

for all $n, 1$ and for all $x ; t, 0$. We have, for $n, 1$,

$$
\begin{aligned}
& E\left[g\left(g\left(t_{i} T_{n} ; q\left(T_{n} ; x\right)+Y_{n}\right)\right): T_{n} \cdot t<T_{n+1}\right], \\
= & e^{i \cdot t}{ }_{1} 0_{t} \frac{(, s)^{n i 1}}{(n ; 1) !} g(q(t ; s ; q(s ; x)+y)) d s \stackrel{o}{(n d y):}
\end{aligned}
$$

Hence we have

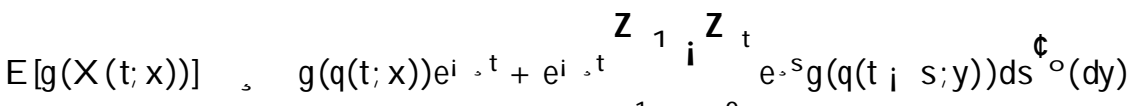

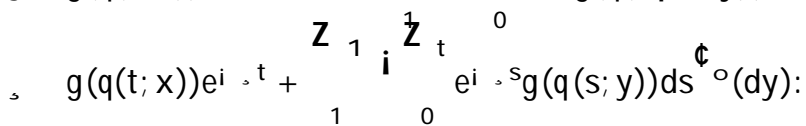

Hence we have the lemma. 2

F irst, we give suф cient conditions for non-existence of moments.

Theorem 3.1 Let $g$ be a nonnegative and nondecreasing function de..ned on $[0 ; 1)$. If there is $v>0$ such that

$$
Z_{1}^{Z_{1} i_{y}} \underset{q(v ; y)}{\frac{g(z)}{r(z)} d z} \stackrel{\Phi}{\Phi}(d y)=1 ;
$$

then

$$
E[g(X(t ; x))]=1 \text { for all } x, 0 \text { and for all } t, v \text {. }
$$


Proof We have, by Lemma 3.1, for any $x, 0$,

$$
\begin{aligned}
& E[g(X(t ; x))], E\left[g\left(X_{1}^{1}\left(t ; X_{1}\right)\right]\right. \\
& e^{i, t_{1}}{ }_{1}^{i^{2} y} \frac{g(z)}{r(z)} d z \stackrel{\phi}{r}(d y)=1
\end{aligned}
$$

for $t, v$. Here we used the change of variable $z=q(s ; y) .2$

Remark 3.1 In the conclusion of Therem 3.1, we can not substitute "for $t, v$ " to "for all $t>0$ ". We give a counter example. Let $r(y) \cdot r$ for $y, 0, o(d y)=\frac{e^{i} y}{y} d y$ for $y>0$ and

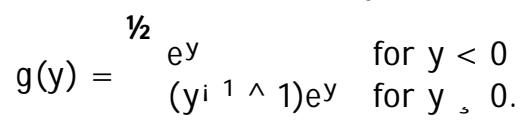

Note that

$$
x i r t+A(t) \cdot x(t ; x) \cdot x+A(t):
$$

The distribution $P(t ; d y)$ of $x_{i} r t+A(t)$ is $\frac{1}{i(t)}(y i x+r t)^{t_{i}{ }^{1}} e^{i(y i x+r t)} d y$. Hence

$$
\begin{aligned}
& Z_{0}^{Z_{1}} g(y) P(t ; d y)=\frac{1}{i(t)}_{x_{Z^{r t}}^{r t}}^{Z_{1}} g(y)(y i x+r t)^{t_{i}{ }^{1}} e^{j(y i x+r t)} d y
\end{aligned}
$$

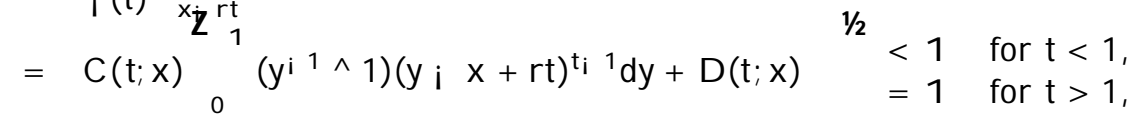

where $0<C(t ; x)<1$ and $0 \cdot D(t ; x)<1$ for every $t, 0$. Then by (2), we have

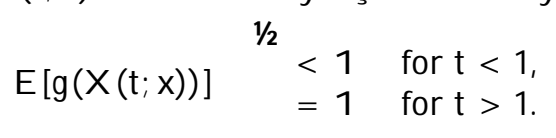

Theorem 3.1 may not be suitable for direct application due to the necessity of computing $q(s ; y)$ in order to check that the condition is valid. One can simplify the above restriction if more conditions are assumed like the following corollaries.

Corollary 3.2 Assume that $r(y)=O(y)$ as $y ! 1$ and $g$ is a nonnegative and nondecreasing function on [0; 1 ) such that, for any $0<a \cdot 1$ and $y>0, g(a y), d(a) g(y)$ with $d(a)>0$. If for

$$
\begin{aligned}
& \mathrm{Z}_{1} \\
& 1 \\
& g(y) \stackrel{\circ}{(d y)}=1
\end{aligned}
$$

then, $E[g(X(t ; x))]=1$ for all $x, 0 ; t>0$.

Proof Note that

$$
Z_{1} \frac{1}{r(y)} d y<1 \quad \text { if and only if } q(t ; 1)=\lim _{y ! 1} q(t ; y)<1 \text { for all } t>0 \text { : }
$$

By the assumption, there is $M>0$ and $y_{0}$ such that

$$
\frac{1}{r(y)}, \frac{1}{M y}
$$


for $y, y_{0}$. Hence $\lim _{y !} \quad q(t ; y)=1$ for any $t>0$. Fix $t_{0}>0$ arbitrarily. Choose $y_{1}>0$ so that $q\left(t_{0} ; y_{1}\right), y_{0}$. Note that $q(t ; y), y_{0}$ for $y, y_{1}$ and $t \cdot t_{0}$. By the de..nition of $q(t ; y)$, we have

$$
t=Z_{\mathrm{q}(\mathrm{t} ; \mathrm{y})}^{\mathrm{Z}_{\mathrm{y}}} \frac{1}{\mathrm{r}(\mathrm{z})} \mathrm{dz}, \frac{1}{\mathrm{M}}_{\mathrm{q}(\mathrm{t} ; \mathrm{y})}^{\mathrm{Z}_{\mathrm{y}}} \frac{1}{\mathrm{z}} d \mathrm{z}=\frac{1}{\mathrm{M}} \log \frac{\mathrm{y}}{\mathrm{q}(\mathrm{t} ; \mathrm{y})}
$$

for $y, y_{1}$ and $t$. $t_{0}$. Hence, $q(t ; y)$, yei $e^{m t}$ for all $t 2\left[0 ; t_{0}\right]$ and all $y, y_{1}$. We have, by the assumption,

$$
Z_{y} \frac{g(z)}{r(t ; y)} d z, g(q(t ; y)) Z_{q(t ; y)} \frac{1}{r(z)} d z, t c\left(e^{i M t}\right) g(y)
$$

for $y, y_{1}$ and $0 \cdot t \cdot t_{0}$. Hence

$$
\underbrace{Z_{1} i_{y}^{Z_{y}}}_{1} \frac{g(z)}{r(z)} d z^{\phi} \underline{o}(d y), t d\left(e^{i M t}\right)_{y_{1}}^{Z_{1}} g(y) o(d y)=1
$$

for $0<\mathrm{t} \cdot \mathrm{t}_{0}$. Then, by Theorem 3.1,

$$
E[g(X(t ; x))]=1 \quad \text { for all } x, 0 \text { and for all } t>0.2
$$

Example 3.1 Let $r(y)=c y^{\circledR}(\circledR \cdot 1)$ and $g(y)=y^{-}\left({ }^{-}>0\right)$. If ${ }_{1}^{R_{1}} y^{-} \varrho(d y)=1$, then $E\left(X(t ; x)^{-}\right)=1$ for $\mathrm{x}, 0 ; \mathrm{t}>0$ by Corollary 3.2 .

Corollary 3.3 Assume that $R_{1} \frac{1}{r(y)} d y<1$ and $g$ is a nonnegative and nondecreasing function on $[0 ; 1)$. If

$$
Z_{1} i_{1}^{Z_{y}} \frac{g(z)}{r(z)} d z \stackrel{o}{o}(d y)=1 \text {; }
$$

then

$$
E[g(X(t ; x))]=1 \text { for all } x, 0 ; t>0 .
$$

Proof Note that due to (3), we have that $q(t ; 1)<1$ for any $t>0$. Without loss of generality we assume that $q(t ; 1)>1$. Then for $y>q(t ; 1)$

$$
Z_{y} \frac{g(z)}{r(z)} d z, \quad Z_{y}(t ; 1) \frac{g(z)}{r(z)} d z:
$$

Then conclusion holds by Theorem 3.1. 2

Example 3.2 Let $r(y)=y^{\circledR}\left({ }^{\circledR}>1\right), g(y)=y^{-}\left({ }^{-}>0\right)$ and ${ }^{-}>{ }^{\circledR} i$ 1. If

$$
Z_{1} y^{-i}{ }^{\circledR+1} \underline{o}(d y)=1 \text {; }
$$

then

$$
E\left[X(t ; x)^{-}\right]=1 \text { for all } x, 0 ; t>0
$$

by Corollary 3.3. 


\section{Existence of moments}

So far, we have studied suఢ cient conditions for non-existence of moments of $X(t)$. Now, we give suf..cient conditions for the existence of moments. Let $x_{0}, 0$. We say that a function $g$ on $\left[x_{0} ; 1\right)$ is submultiplicative on $\left[x_{0} ; 1\right)$ if it is nonnegative and there is a constant $a>0$ such that

$$
g(y+z) \cdot \operatorname{ag}(y) g(z) \text { for } y ; z, x_{0}:
$$

Lemma 4.1 Suppose that $g(y)$ is a nonnegat jye and nondecreasing function on $[0 ; 1$ ) which is submul-

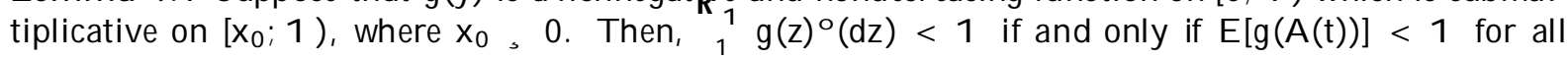
$t, 0$.

Proof Under the assumption of the lemma, necessary facts for the proof of Theorem 25.3 of [12] hold. 2

Theorem 4.1 Let $x_{0}, 0$ and let $9(y)$ be a nonnegative and nondecreasing function on $[0 ; 1)$ which is submultiplicative on $\left[x_{0} ; 1\right)$. Then $\underset{1}{1} g(z) \circ(d z)<1$ implies

$$
E[g(X(t ; x))]<1 \text { for all } x, 0 ; t>0 .
$$

Proof Note that $X(t ; x) \cdot x+A(t)$ for all $t, 0$. By Lemma 4.1,

$$
E[g(A(t))]<1:
$$

This yields the conclusion. 2

Example 4.1 L et $g(y)=y^{-}$with $^{-}>0$ and $r$ satisfying $(\mathrm{H} 0)$. If

$$
Z_{1} y^{-} \circ(d y)<1 \text {; }
$$

then, by Theorem 4.1, $E\left[X(t ; x)^{-}\right]<1$ for all $x, 0 ; t>0$.

In the case $r(y)=y^{\circledR}, \circledR>1$, conditions weaker than (4) are suф cient for the existence of moments of $f X(t) g$ of order ${ }^{-}$. Next three theorems treat this case.

Theorem 4.2 A ssume that $\stackrel{o}{(}(0 ; 1))<1$. Let $\mathrm{g}$ be a nonnegative and nondecreasing function on $[0 ; 1)$. Suppose that there is $x_{0}>0$ and $C, 0$ such that $G(y)={ }_{x_{0}} \frac{g(z)}{r(z)} d z+C$ is submultiplicative on $\left[\mathrm{x}_{0} ; 1\right)$. Then

$$
Z_{1} G(y) \circ(d y)<1
$$

implies

$$
E[g(X(t ; x))]<1 \text { for all } x, 0 ; t>0 \text { : }
$$

Proof Let, $=o((0 ; 1))$ and $F(d y)=\underline{1} o(d y)$. We de.ne $f_{\iota_{k}} g, f Y_{k} g, f T_{k} g$ as Section 2 for $f X(t) g$. Choose $x$ so that $x>x_{0}$. Note that

$$
E\left[g(X(t ; x)): 0 \cdot t<T_{1}\right]=g(q(t ; x)) e^{i, t}
$$


and, for $\mathrm{n}, 1$,

$$
\begin{aligned}
& E\left[g(X(t ; x)): T_{n} \cdot t<T_{n}+i_{n+1}\right] \\
& =E\left[g\left(q\left(t ; T_{n} ; X\left(T_{n} ; ; x\right)+Y_{n}\right)\right): T_{n} \cdot t<T_{n}+i_{n+1}\right]
\end{aligned}
$$

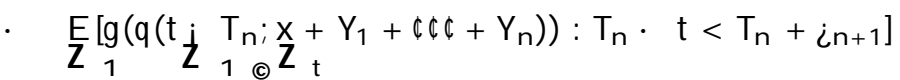

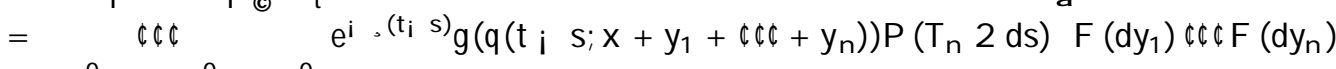

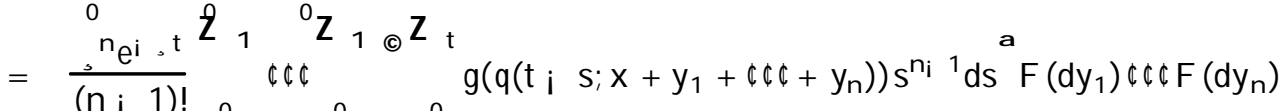

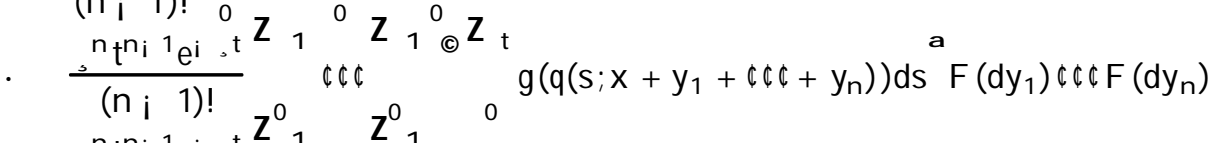

$$
\begin{aligned}
& =\frac{{ }_{n} t^{n i 1} e^{i}, t}{(n i 1) !} Z_{1}^{0} Z_{1}^{0} Z_{0}
\end{aligned}
$$

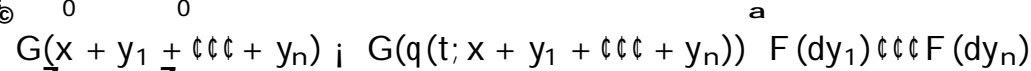

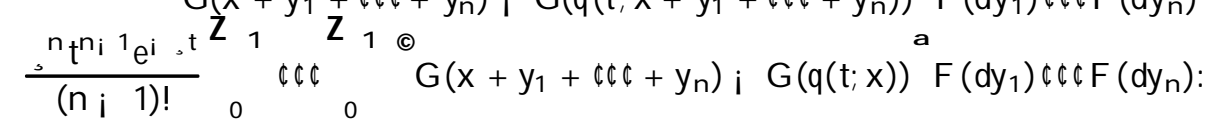

Since $G(y)$ is submultiplicative on $\left[x_{0} ; 1\right)$, there is $c>0$ such that

$$
G\left(x+y_{1}+\not \notin \Phi+y_{n}\right) \cdot c^{n_{i}{ }^{1}}{ }_{i=1}^{n} G\left(x+y_{i}\right):
$$

Also by the submultiplicativity,

$$
{ }_{0}^{Z_{1}} G(x+y) F(d y) \cdot{ }_{0}^{Z_{x_{0}}} G(x+y) F(d y)+c G(x)_{x_{0}}^{Z_{1}} G(y) F(d y)=: K<1:
$$

Then, we have

$$
E\left[g(X(t ; x)): T_{n} \cdot t<T_{n}+i_{n+1}\right] \cdot \frac{{ }^{n} t^{n_{i}}{ }^{1} e^{i}, t}{(n ; 1) !} f K(d K)^{n_{i} 1} i G(q(t ; x)) g:
$$

Hence,

$$
\begin{aligned}
E[g(X(t ; x))] & \cdot g(q(t ; x)) e^{i, t}+{ }_{n=1}^{X} \frac{(, t)^{n_{i} 1}}{(n ; 1) !} e^{i, t} f K(c K)^{n_{i} 1} ; G(q(t ; x)) g \\
= & g(q(t ; x)) e^{i, t}+, K e^{t(c K i 1)} i, G(q(t ; x)):
\end{aligned}
$$

To prove the ...nit eness of the above expectation is enough to prove that $G(q(t ; x))$ can not take the value $i 1$. This is only possible if $q(t ; x)=0$. In such a case ${ }_{0}^{x} \frac{1}{r(z)} d z<1$ which implies that $G(0)>i 1$. Therefore $E[g(X(t ; x)))<1$ for $x, x_{0}$. To conclude for $x<x_{0}$ is enough to note that $X(t ; x) \cdot X\left(t ; x_{0}\right)$ for $0 \cdot \mathrm{x}<\mathrm{x}_{0}: 2$

In the previous result we had to assume that the Lévy measure was ..nite, in the next we exchange this condition with a restriction on $r$ and further restriction on $g$.

Theorem 4.3 A ssume that $r$ is nondecreasing. Let $g$ be a nonnegative and nondecreasing on $[0 ; 1)$. Suppose that there is $x_{0}>0$ and $C, 0$ such that both $g(y)$ and $G(y)={ }_{x_{0}} \frac{g(z)}{r(z)} d z+C$ are submultiplicative on $\left[\mathrm{x}_{0} ; 1\right)$. Then

$$
Z_{1} G(y) \stackrel{o}{ }(d y)<1
$$


implies

$$
E[g(X(t ; x))]<1 \text { for } x, 0 ; t>0 \text {. }
$$

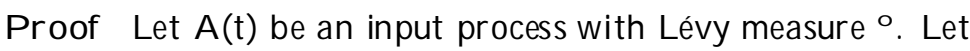

$$
A_{1}(t)={ }_{s \cdot t}^{X} f(s) ; A(s i) g l_{f A(s) ; A}\left(s_{i}\right)>1 g
$$

and let $X_{1}(t ; x)$ be the storage process starting at $x$ with input process $A_{1}(t)$. Then $f X_{1}(t ; x) g$ satis.. es

$$
X_{1}(t ; x)=x_{i}{ }_{0}^{Z_{t}} r\left(X_{1}(s ; x)\right) d s+A_{1}(t):
$$

Since $X(t ; x), X_{1}(t ; x)$ and $r$ is nondecreasing, we have

$$
\begin{aligned}
X(t ; x) ; X_{1}(t ; x) & =i_{t}^{Z_{t}} f r(X(s ; x)) ; r\left(X_{1}(s ; x)\right) g d s+A(t) ; A_{1}(t) \\
& \cdot A(t) ; A_{1}(t):
\end{aligned}
$$

By the submultiplicativity and nondecreasingness of $g$, we have

$$
\begin{aligned}
E[g(X(t ; x))] \cdot & E\left[a g\left(x_{0}+X(t ; x) ; X_{1}(t ; x)\right) g\left(x_{0}+X_{1}(t ; x)\right)\right] \\
\cdot & E\left[a g\left(x_{0}+A(t) ; \quad A_{1}(t)\right) g\left(x_{0}+X_{1}(t ; x)\right)\right] \\
= & a E\left[g\left(x_{0}+A(t) ; \quad A_{1}(t)\right)\right] E\left[g\left(x_{0}+X_{1}(t ; x)\right)\right]
\end{aligned}
$$

where $a>0$ is the constant of submultiplicativity of $\mathrm{g}$. Here, we also used the mutual independence of $A_{i} A_{1}$ and $X_{1}$. By Lemma 4.1 (note that the Lévy process $A_{i} A_{1}$ has a Lévy measure with bounded support),

$$
E\left[g\left(x_{0}+A(t) ; A_{1}(t)\right)\right]<1 \quad \text { for } t>0 .
$$

Now we prove the ..niteness of $E\left[g\left(x_{0}+X_{1}(t ; x)\right)\right]$. If $X_{1}(t ; x) \cdot x_{0}$, then $g\left(x_{0}+X_{1}(t ; x)\right) \cdot g\left(2 x_{0}\right)$. If $X_{1}(t ; x)>x_{0}$ then $g\left(x_{0}+X_{1}(t ; x)\right) \cdot \operatorname{ag}\left(x_{0}\right) g\left(X_{1}(t ; x)\right)$. In any case,

$$
g\left(x_{0}+X_{1}(t ; x)\right) \cdot g\left(2 x_{0}\right)+\operatorname{ag}\left(x_{0}\right) g\left(X_{1}(t ; x)\right)
$$

By the preceding Theorem, we have

$$
E\left[g\left(x_{0}+X_{1}(t ; x)\right)\right]<1 \text { for all } t>0:
$$

We get the conclusion. 2

If we assume convexity of $r$, condition on $g$ for the existence of $g$-moment of $X$ becomes quit simple. In order to show this, we prepare two lemmas.

Lemma 4.2 If $r$ is convex on $[0 ; 1)$, then $q(t ; y+z) \cdot q(t ; y)+q(t ; z)$ for all $t ; y ; z, 0$.

Proof Since $r$ is convex on $[0 ; 1)$ and $r(0)=0, \quad R_{y} \frac{1}{r(z)} d z=1$ for all $y>0$. Then

$$
Z_{y(t ; y)} \frac{1}{r(z)} d z=t
$$


for all $y>0$ and $t, 0$. Hence $q(t ; y)$ is left dixerentiable in $y>0$ and the left derivative $\frac{\varrho}{\varrho} q(t ; y)$ satis..es the equation

$$
\frac{1}{r(y)} i \frac{1}{r(q(t ; y))} \stackrel{@}{@} q(t ; y)=0:
$$

Hence

$$
\frac{@}{@ y} q(\mathrm{t} ; \mathrm{y})=\frac{\mathrm{r}(\mathrm{q}(\mathrm{t} ; \mathrm{y}))}{\mathrm{r}(\mathrm{y})} \text { : }
$$

Since $r$ is convex, $r$ has a nondecresing left derivative $r^{x}$. Hence $\frac{d}{\varrho} q(t ; y)$ has a left derivative which satis.es

$$
\left(\frac{@}{@} q(t ; y)\right)^{\circledR}=r(q(t ; y)) \frac{r^{x}(q(t ; y)) ; r^{x}(y)}{r(y)^{2}} \cdot 0 \text { a.e. } y>0 \text {. }
$$

Here, we used $q(t ; y) \cdot y$. Hence $q(t ; y)$ is concave in $y$. Since

$$
q(t ; y+z) i q(t ; y)={ }_{0}^{z} \frac{@}{@ u} q(t ; y+u) d u \text {; }
$$

we have

$$
q(t ; y+z) ; q(t ; y) i q(t ; z)=\int_{0}^{z_{z}} f \frac{@}{@ u} q(t ; y+z) i \frac{@}{@ u} q(t ; u) g d u
$$

for all $y ; z ; t, 0$, by concavity of $q .2$

Remark 4.1 Assume that $r$ is convex on $[0 ; 1)$. L et $z(t)$ be a nonnegative nondecreasing st ep function on $[0 ; 1)$. De..ne $x(t ; z)$ by

$$
x(t ; z)=i_{0}^{Z_{t}} r(x(s ; z)) d s+z(t):
$$

Let $z_{0}(t)=x$ and $z_{1}(t)=z_{1} 1_{\left[t_{1} ; 1\right.}$ ) (t) for $x ; z_{1}, 0$ and $t 2[0 ; 1)$. Then, by Lemma 4.2,

$$
\mathrm{x}\left(\mathrm{t} ; \mathrm{z}_{0}+\mathrm{z}_{1}(\phi)=\mathrm{q}\left(\mathrm{t} ; \mathrm{t}_{1} ; \mathrm{q}\left(\mathrm{t}_{1} ; \mathrm{x}\right)+\mathrm{z}_{1}\right) \cdot \mathrm{q}(\mathrm{t} ; \mathrm{x})+\mathrm{q}\left(\mathrm{t}_{\mathrm{i}} \mathrm{t}_{1} ; \mathrm{z}_{1}\right)=\mathrm{x}\left(\mathrm{t} ; \mathrm{z}_{0}\right)+\mathrm{x}\left(\mathrm{t} ; \mathrm{z}_{1}\right)\right.
$$

for $t, t_{1}$. The above inequality also holds for $t<t_{1}$. In the same way, for nonnegative and nondecreasing step functions $z_{1}(t)$ and $z_{2}(t)$ with ..nite steps up to $t$, we have

$$
x\left(t ; z_{1}+z_{2}\right) \cdot x\left(t ; z_{1}\right)+x\left(t ; z_{2}\right):
$$

Taking a limit, we have the above inequality for all nonnegative and nondecreasing step functions $z_{1}(t)$ and $z_{2}(t)$. This shows that the storage process $X(t ; x)$ is a subadditive functional of $X+A$ provided that $r$ is convex on [0; 1 ) ([11]).

Let $Y(t ; x)$ be a nonnegative random variable with Laplace transform

$$
E[\operatorname{expf} i \mu Y(t ; x) g]=\exp \left[i \mu q(t ; x)+{ }_{0}^{Z_{1}} f_{0}^{Z_{t}}\left(e^{j \mu(s ; y)} i 1\right) d s g^{\varrho}(d y)\right]:
$$

The integral of the right side of the above equality is well de..ned since $q(t ; y) \cdot y$. It may be interesting that $Y(t ; x)$ is represented as

$$
\begin{aligned}
Y(t ; x) & =q(t ; x)+Z_{Z^{(0 ; t]}}^{Z} q(t ; s ; d A(s)) \\
& =q(t ; x)+{ }_{[0 ; t] f(0 ; 1)} q(s ; y) N(d s d y)
\end{aligned}
$$


in law. Here,

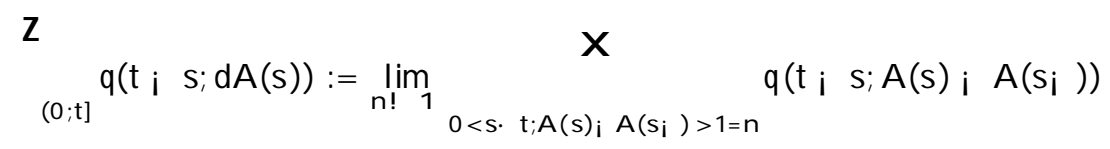

and $\mathrm{N}$ i $\mathrm{R}_{\mathrm{R}}$ a Poisson random measure on $[0 ; \mathrm{t}] \mathrm{f}(0 ; 1)$ with intensity measure $d s^{\circ}(\mathrm{dy})$. The stochastic $\mathrm{R}^{\text {integral }}{ }_{(0 ; t]} \mathrm{q}\left(\mathrm{t}_{i} \mathrm{~s} ; \mathrm{dA}(\mathrm{s})\right)$ can be regarded as a natural extension of the stochastic integral representation ${ }_{(0 ; t]} \mathrm{e}^{\mathrm{a}\left(\mathrm{t}_{i} \mathrm{~s}\right)} \mathrm{dA}(\mathrm{s})$ of process of Ornstein-Uhlenbeck type (that is when $r(y)=a y$, se [13]).

For two random variables $X ; Y$, it is said that $X, Y$ in stochastic ordering sense if $P(X>y)$. $P(Y>y)$ for all y $([5])$.

Lemma 4.3 If $r$ is convex on $[0 ; 1)$, then

$$
X(t ; x) \cdot Y(t ; x)
$$

for all $x ; t, 0$ in stochastic ordering sense.

Proof First, we assume that, $=o((0 ; 1))<1$. Let $N_{t}$ be a Poisson process with intensity,. Since $X$ is a subadditive functional of $X+A$, we have that

$$
\begin{aligned}
X(t ; x) & =q(t ; x)+q\left(t ; T_{1} ; Y_{1}\right)+\phi \notin \Phi+q\left(t ; T_{N_{t}} ; Y_{N_{t}}\right) \\
= & q(t ; x)+{ }_{[0 ; t] \notin(0 ; 1)} q(t ; s ; y) N(d s d y):
\end{aligned}
$$

Now, assume that,$=1$. Let $X_{n}(t ; x)$ be a storage process de. ned in Section 2 via $A_{n}(t)$. Then

$$
X_{n}(t ; x) \cdot q(t ; x)+Z_{[0 ; t] f(1=n ; 1)} q(s ; y) N(d s d y)
$$

in stochastic ordering sense, $X_{n}(t ; x) " X(t ; x)$ and

$$
q(t ; x)+Z_{[0 ; t] f(1=n ; 1)}^{Z} q(s ; y) N(d s d y) " q(t ; x)+Z_{[0 ; t] f(0 ; 1)}^{Z} q(s ; y) N(d s d y)
$$

a.s. as $\mathrm{n} ! 1$. Hence $X(t ; \mathrm{X}) \cdot \mathrm{Y}(\mathrm{t} ; \mathrm{x})$ in stochastic ordering sense. 2

Theorem 4.4 A ssume that $r$ is convex on $[0 ; 1)$. Let $g$ be a nonnegative and nondecreasing function on $[0 ; 1)$ such that $g$ is submultipicative on an interval $\left[x_{0} ; 1\right),\left(x_{0}>0\right)$. If

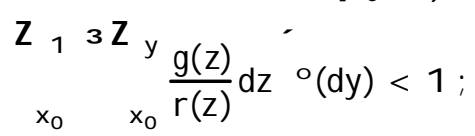

then

$$
E[g(X(t ; x))]<1 \quad \text { for all } x, 0 ; t>0:
$$

Proof We de..ne $z(t ; x)$ by

$$
z(t ; x)=\operatorname{supf} z>0: \quad z_{x} \frac{1}{r(u)} d u \cdot \operatorname{tg}
$$


for $x>0$. If (7) holds, then

$$
\begin{aligned}
& \mathrm{Z}_{1}{ }_{3} \mathrm{Z}_{\mathrm{t}} \\
& \text { If } q(s ; y), \quad x_{0} g g(q(s ; y)) d s \stackrel{o}{(d y)}
\end{aligned}
$$

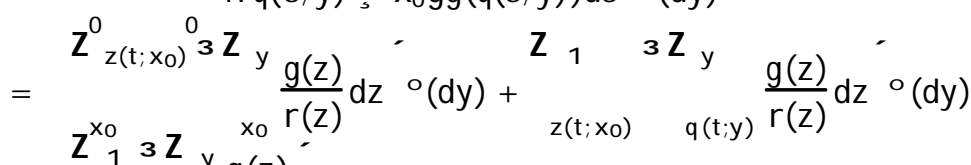

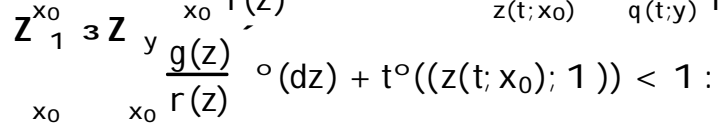

Hence by Lemma 4.1 and 4.3, we get (8). 2

Example $4.2 \mathrm{~L}$ et $g(y)=y^{-}$and $r(y)=y^{\circledR}\left(\circledR>1 ;^{-}>0\right)$. T hen by $T$ heorem 4.3 or 4.4 we have that in the following cases $E\left[X(t ; x)^{-}\right]<1$ for all $x, 0 ; t>0$ :
(a) If ${ }^{-}<\otimes_{i} 1$.
(b) If ${ }^{-}=\mathbb{B}_{\mathrm{i}} 1$ and $\mathrm{R}_{1} \log \mathrm{y}^{\mathrm{o}}(\mathrm{dy})<1$.
(c) If ${ }^{-}>\circledR_{\mathrm{i}} 1$ and ${ }_{1} \mathrm{y}^{-i}{ }^{\circledR+1} \underline{0}(\mathrm{dy})<1$.

\section{Examples}

In all the previous examples we always used power functions. Here we exhibit functions $\mathrm{g}$ and $\mathrm{G}$ which are not power functions and satisfy the assumptions of previous T heorems and Corollaries.

Example $5.1 \mathrm{~L}$ et $\mathrm{g}(\mathrm{y})$ be a function of the form

$$
g(y)=d y) \operatorname{expf}_{1}^{Z_{y}} \frac{2(u)}{u} d u g ;
$$

where $0<c_{1} \cdot c(y) \cdot c_{2}$ and $0<{ }^{2}(y) \cdot c_{3}$ for all $y, 0$.

(a) For $0<a<1$ and $y>0$,

$$
\frac{g(a y)}{g(y)}=\frac{c(a y)}{c(y)} \operatorname{expf}_{i} \quad Z_{y} \frac{2(u)}{u} \text { dug, } \frac{c_{1}}{c_{2}} a^{c_{3}}:
$$

Hence $g(\Phi$ satis..es the assumption of Corollary 3.2 provided that $c(\Phi$ is nondecreasing.

(b) Suppose additionally that $\frac{2(u)}{u}$ is nonincreasing on $[1 ; 1)$, then, for $y ; z, 1$,

$$
\begin{aligned}
& g(y+z)=c(y+z) \operatorname{expf}{ }_{1}^{Z_{y+z}} \frac{2(u)}{u} d u g \\
& =\frac{d y+z)}{d y) c(z)} g(y) g(z) \operatorname{expf}\left(Z_{y+z} Z_{z}{ }_{1}\right) \frac{{ }^{2}(u)}{u} d u g \\
& =\frac{d y+z)}{d y) c(z)} g(y) g(z) \operatorname{expf} z_{z+1}^{y} \frac{2(w+y i 1)}{w+y i 1} d w i_{1} \frac{z^{2}(u)}{u} d u g \\
& \text { - } \frac{d y+z)}{d y) c(z)} g(y) g(z) \operatorname{expf}_{z}^{z^{1}} \frac{2(u)}{u} d u g \\
& \text { - } \frac{2^{c_{3}} c_{2}}{\left(c_{1}\right)^{2}} g(y) g(z) \text {; }
\end{aligned}
$$

that is, $\mathrm{g}$ is submultiplicative on $[1 ; 1)$. Hence $\mathrm{g}(\mathrm{y})$ satis..es the assumption of $\mathrm{T}$ heorem 4.1 provided that $c(\phi$ is nondecreasing. 
Example 5.2 L et $c>0$ and ${ }^{\circledR}>0$ betwo ..xed constants. Let ${ }^{2}(y)$ be o nonnegative and nondecreasing function on $[1 ; 1)$ such that $\lim _{y} !{ }_{1}{ }^{2}(y)={ }^{-}>0,2(1)+1 ; \quad \circledast>0$ and ${ }_{1} \frac{{ }^{-} i^{2}(u)}{u} d u<1$. De.ne $g$ and G by

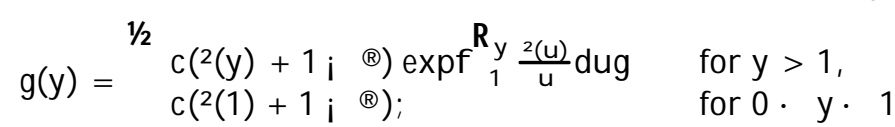

and

$$
G(y)={ }_{1}^{Z_{y}} z^{i}{ }^{\circledR} g(z) d z \text { for } y, 1,
$$

respectively. Then,

$$
G(y)=\operatorname{cexpf}_{1}^{Z_{y}} \frac{{ }^{2}(u) i \circledast+1}{u} \operatorname{dug} i c \text { for } y, 1 \text { : }
$$

The function $g$ is nonnegative and nondecreasing on $[0 ; 1)$. Let $y ; z, 1$. Then,

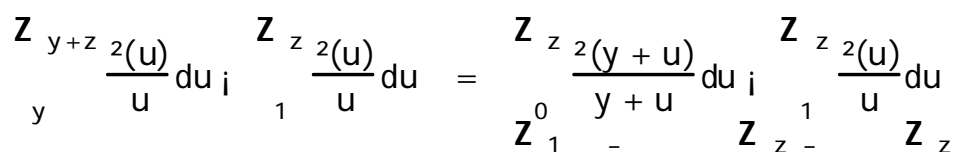

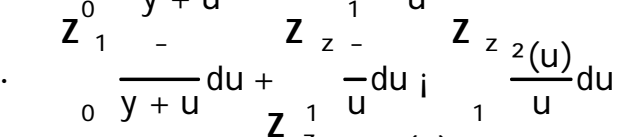

$$
\begin{aligned}
& =-\log \frac{y+1}{y_{z_{1}}}+{ }^{-1} \frac{{ }^{1}{ }^{2}(u)}{u} d u \\
& -\log 2+{ }_{1} \frac{Z_{1}{ }^{2}(u)}{u} d u<1:
\end{aligned}
$$

Repeating similar calculations as in example 5.1 we obtain the submultiplicativity of $g(y)$ on $[1 ; 1)$. In the same way, we have the submultiplicativity of $G(y)$ on $[1 ; 1)$. Hence $g(y)$ satis..es the assumptions in Theorems 4.3 and 4.4 with $x_{0}=1$ and $r(y)=y^{\circledR}$.

Examples 5.1 and 5.2 are slight departures of functions $g$ that are of polynomial type. The following introduces a similar study for exponential type functions.

Example 5.3 Let $r(y)=y^{\circledR}\left({ }^{\circledR}>0\right)$. Then, $g(y)=y^{\circledR} e^{a y},(a>0)$, satis..es the assumption of Theorem 4.1 with $x_{0}=1$. Also, $G(y)=a^{i}{ }^{1} e^{a y}$ satis..es the assumption of Theorem 4.3 and Theorem 4.4 with $x_{0}=1$. Here one can also extend these examples to generate a similar class as in Examples 5.1 and 5.2. In fact, if $g(y)=c(y) \exp \left({ }_{1}^{y} f(u) d u\right)$ for a submultiplicative function $c$ and a positive function $f$ nonincreasing and bounded then $g$ is submultiplicative in $[1 ; 1)$. Similarly, one has that if $g(y)=\left(f(y) ; \frac{\otimes}{y}\right) \exp \left({ }_{1} f(u) d u\right)$ for $f(y) ; \frac{\otimes}{y}$ bounded above and below by positive constants then $g$ and $\mathrm{G}$ are submultiplicative functions in $[1 ; 1)$.

\section{Tail probability}

In this section, we discuss tail probabilities of storage processes.

Lemma 6.1 For all $x ; t, 0$,

$$
P(X(t ; x)>y) \cdot P(x+A(t)>y):
$$

Proof It is obvious by the inequality $X(t ; x) \cdot x+A(t) .2$ 
Lemma 6.2 If $r$ is concave on $(0 ; 1)$, then

$$
q(t ; y+z), q(t ; y)+q(t ; z)
$$

for all $y ; z>0$ and $t, 0$.

Proof We have

$$
Z_{y(t ; y)} \frac{1}{r(z)} d z=t \quad \text { for } y>z(t ; 0+) \text {. }
$$

As in the proof of Lemma 4.2,

$$
q(t ; y+z) \text { i } q(t ; y) ; q(t ; z), 0
$$

for $y ; z>z(t ; 0+)$ and $t, 0$. If $0 \cdot y \cdot z(t ; 0+)$, then

$$
q(t ; y)+q(t ; z)=q(t ; z) \cdot q(t ; y+z)
$$

by nondecreasingness of $\mathrm{q}(\mathrm{t} ; \mathrm{y})$ in $\mathrm{y}, 0.2$

Lemma 6.3 If $r$ is concave on $(0 ; 1)$, then

$$
X(t ; x), Y(t ; x)
$$

in stochastic ordering sense for all $x ; t, 0$, where $Y(t ; x)$ is de. ned by (6).

Proof The proof is accomplished using the same argument as the proof of Lemma 4.3 using Lemma 6.2. 2

Let $Q(y)={ }_{0}^{R_{1}}\left(\begin{array}{c}R_{t} \\ 0\end{array} 1_{(y ; 1)}(q(s ; z)) d s-(d z)\right.$ for $y>0$.

Lemma 6.4 (a) If $r$ is bounded and $\varrho((y+z ; 1)) \bumpeq((y ; 1)) ! 1$ as $y$ ! 1 for every $z, 0$, then

$$
\mathrm{Q}(\mathrm{y}) \gg \mathrm{to}((\mathrm{y} ; 1))
$$

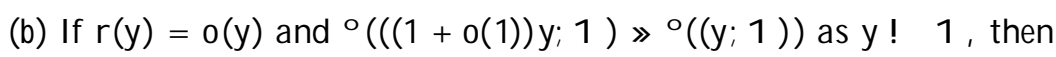

$$
\mathrm{Q}(\mathrm{y}) \gg \mathrm{to}((\mathrm{y} ; 1))
$$

(c) If $r(y)=O(y)$ as $y$ ! 1 and $o((y ; 1))$ is slowly varying at in..nity, then

$$
\mathrm{Q}(\mathrm{y}) \gg \mathrm{t}-((\mathrm{y} ; 1)) \text { : }
$$

(d) If $r(y) »$ ay as $y$ ! 1 and $\cong((y ; 1))=y^{i}{ }^{-} L(y),\left(a ;^{-}>0\right)$ where $L(y)$ is slowly varying at in..nity, then

(e) If $\begin{gathered}R_{1} \\ 1\end{gathered} \frac{1}{r(y)} d y<1$, then

$$
\begin{aligned}
& Q(y) » \frac{1_{i} e^{i a^{-t}}}{a^{-}} y^{i^{-}} L(y): \\
& Q(y){ }_{y}^{Z_{1}} \frac{o((z ; 1))}{r(z)} d z:
\end{aligned}
$$


Proof We have

$$
\begin{aligned}
& \mathrm{Q}(\mathrm{y})=\mathrm{Z}_{1} \mathrm{i}^{\mathrm{Z}_{\mathrm{t}}} 1 \mathrm{fq}(\mathrm{s} ; \mathrm{z})>\mathrm{ygds}^{\phi_{\mathrm{o}}(\mathrm{dz})}
\end{aligned}
$$

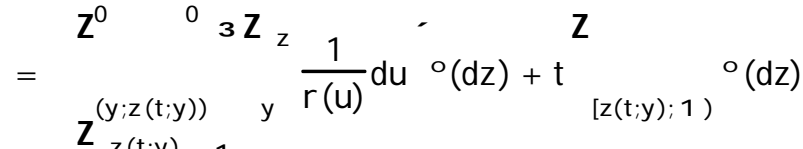

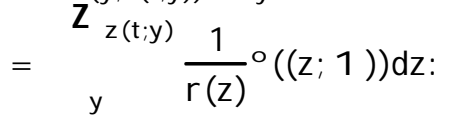

Hence

$$
\text { too ((z(t;y);1 )) } \cdot \mathrm{Q}(\mathrm{y}) \cdot \mathrm{to}((\mathrm{y} ; 1)) \text { : }
$$

Note that in cases (a) 》(d), $\begin{gathered}R_{1} \\ 1\end{gathered} \frac{1}{r(u)} d u=1$. (a) If there is $M>0$ such that $r$. $M$ on $[0 ; 1)$, then

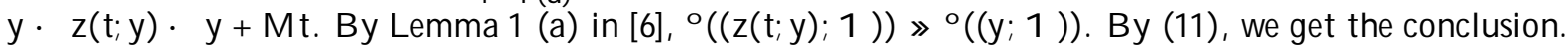
(b) For any ${ }^{2}>0$, there is $z_{0}>0$ such that $r(u)<{ }^{2} u$ for all $u>z_{0}$. Then $\frac{1}{r(u)}>\frac{1}{2 u}$ for all $u>z_{0}$. Hence

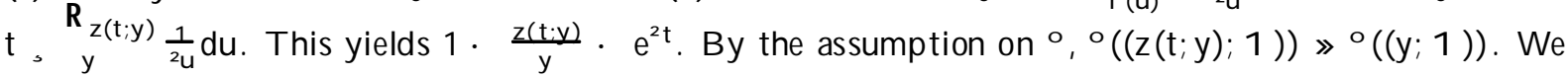
get the conclusion by (11).

(c) There is $M>0$ and $z_{0}>0$ such that

$$
\frac{1}{\mathrm{r}(\mathrm{u})}, \frac{1}{\mathrm{Mu}}
$$

for $u, z_{0}$. By the argument in the proof of $(a), \frac{z(t ; y)}{y} \cdot e^{M t}$. Since $\varrho$ is slowly varying, $\varrho((z(t ; y) ; 1)) »$ $o((y ; 1))$. We get the conclusion by (11).

(d) For any ${ }^{2}>0$, there is $y_{0}>0$ such that $\left(1 ;{ }^{2}\right)$ ay $\cdot r(y) \cdot\left(1+{ }^{2}\right)$ ay for $y>y_{0}$. By (10),

$$
\begin{array}{rl}
\left(1+{ }^{2}\right)^{i}{ }^{Z}{ }^{z(t ; y)} \frac{o((z ; 1))}{a z} d z & Q(y) \\
& \cdot\left(1 ;{ }^{2}\right)^{i}{ }^{Z_{z(t ; y)}} \frac{\underline{o}((z ; 1))}{a z} d z
\end{array}
$$

for $\left.y>y_{0} . A s \stackrel{o}{(}(z ; 1)\right) \gg z^{i}{ }^{-} L(z)$, we have

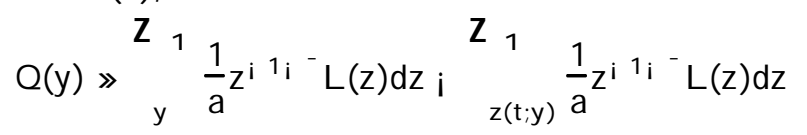

as y! 1. Hence

$$
Q(y) \gg{\frac{1}{a^{-}}}^{3} y^{i-} L(y) ; z(t ; y) i^{-} L(z(t ; y))
$$

as $y ! 1$. Since $z(t ; y) » y e^{\text {at }}$ as $y ! 1$,

$$
Q(y) \gg \frac{1 i e^{a^{a^{-} t}}}{a^{-}} y^{i-} L(y)
$$

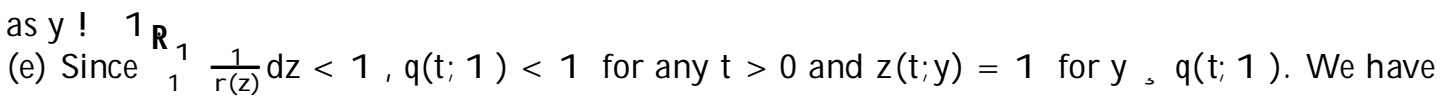

$$
\mathrm{Q}(\mathrm{y})=\mathrm{Z}_{\mathrm{1}} \frac{1}{\mathrm{r}(\mathrm{z})} \mathrm{o}((\mathrm{z} ; 1)) \mathrm{dz} \text { : }
$$


A probability measure $P$ on $[0 ; 1)$ is said to be subexponential ([3]) if

$$
\lim _{y ! 1} \frac{P^{2 x}((y ; 1))}{P((y ; 1))}=2
$$

holds. Note that if $\mathrm{P}((y ; 1))$ is regularly varying at in..nity with nonpositive exponent, then $\mathrm{P}$ is subexponential by Corollary on p. 279 of [7].

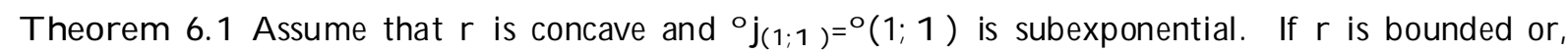

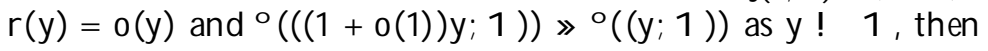

$$
P(X(t ; x)>y) \gg t o((y ; 1))
$$

as $y ! 1$, for all $x ; t, 0$.

Proof Let $f A(t) g$ be an input process of $f X(t ; x) g$. Since $r$ is concave, we have

$$
P(Y(t ; x)>y) \cdot P(X(t ; x)>y) \cdot P(A(t)>y i x):
$$

By Lemma 6.4 (a), (b) and Theorem 1 ([6]), we have

$$
\mathrm{P}(\mathrm{Y}(\mathrm{t} ; \mathrm{x})>\mathrm{y}) \mathrm{t}_{\mathrm{y}}^{\mathrm{Z}_{1}} \stackrel{\mathrm{o}}{ }(\mathrm{dz}):
$$

By Theorem 1 ([6]) and Lemma 1 ([6]), we have

$$
\begin{aligned}
& P(A(t)>y i x) \gg t^{Z_{1}} \stackrel{o}{ }(d z) \\
& Z_{1}^{y i x} \\
& \gg t_{y} \stackrel{o}{(d z)} \text { : }
\end{aligned}
$$

2

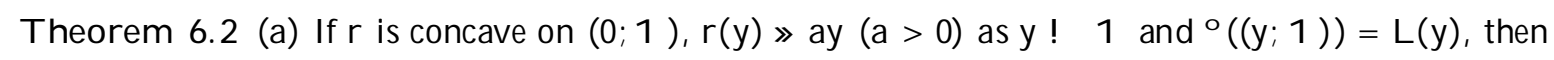

$$
P(X(t ; x)>y) \gg t L(y)
$$

and

(b) if $r$ is convex on $[0 ; 1), r(y) »$ ay and $\cong((y ; 1))=y^{i^{-}} L(y)\left({ }^{-}>0\right)$, then

$$
P(X(t ; x)>y) » \frac{1 i e^{a^{-a t}}}{a^{-}} y^{-} L(y)
$$

as $y$ ! 1 for all $x ; t, 0$. Here $L(y)$ is a function slowly varying at in..nity.

Proof (a) Note that (12) holds. By Lemma 6.4 (c), we have

$$
P(X(t ; x)) \gg t L(y) \text { as } y ! 1 \text {. }
$$

(b) B y Lemma 6.4 (d),

$$
Q(y) \gg \frac{1 ; e^{e^{a^{-} t}}}{a^{-}} y^{-} L(y) \text { as } y ! 1
$$


Let

$$
A_{y}=f(s ; u) 2[0 ; t] f(0 ; 1): x\left(t ; u 1_{[s ; 1)}(\phi)>y g ;\right.
$$

where $x(t ; z)$ is a functional of a nonnegative and nondecreasing step function $z$ de..ned by (5). Note that

$$
\begin{aligned}
& Z Z_{t} \\
& 1_{(0 ; 1)} \quad 0
\end{aligned}
$$

Since $X(t ; x)$ is a subadditive functional of $x+A$ and a probability measure $; Q(d y)=Q(1)$ on $(1 ; 1)$ is subexponential,

$$
P(X(t ; x)>y) \gg Q(y)
$$

as $y$ ! 1 by Theorem 3.1 and Example (Lévy motion) in [11]. We get the conclusion. 2

Theorem 6.3 If $r$ is convex and a probability measure; $Q(d y)=Q(1)$ on $(1 ; 1)$ is subexponential, then

$$
P(X(t ; x)>y) \gg Z_{z} \frac{\left.Z_{z} ; y\right)}{r(z)} d z
$$

as $y$ ! 1 for all $x, 0$ and $t>0$. Under an additional assumption $\mathrm{R}_{1} \frac{1}{r(y)} d y<1$,

$$
P(X(t ; x)>y) Z_{y} \frac{o((z ; 1))}{r(z)} d z
$$

as $y$ ! 1 for all $\mathrm{x}, 0$ and $\mathrm{t}>0$.

Proof As in the proof of Theorem 6.2 (b), we have

$$
P(X(t ; x)>y) \gg Q(y)
$$

as y ! 1 . By (10)

$$
P(X(t ; x)>y){ }_{y}^{Z_{z(t ; y)}} \frac{\underline{o}((z ; 1))}{r(z)} d z
$$

as $y$ ! 1. If ${ }_{1}^{R_{1}} \frac{1}{r(y)} d y<1$, then we have by Lemma 6.4 (e), that

$$
P(X(t ; x)>y) Z_{y} \frac{o((z ; 1))}{r(z)} d z
$$

as y ! 1.2

Remarks

1a. In Theorems 4.2-4.4, assumptions for $r$ can be relaxed as follows: There exists a function $r_{i}$ such that $r_{i} \cdot r$ and the assumptions on $r$ are replaced by the same ones where $r$ is replaced by $r_{i}$.

1b. Additionally, if one assumes that

$$
Z_{1} \frac{\underline{o}((z ; 1))}{r(z)} d z » Z_{1} \frac{o((z ; 1))}{r_{i}(z)} d z
$$

as $y$ ! 1 then $T$ heorem 6.3 is also satis..ed.

2. In Theorem 6.1, assumptions for $r$ can be relaxed as follows: There exists a function $r_{+}$such that $r_{+}, r$ and the assumptions on $r$ are replaced by the same ones where $r$ is replaced by $r_{+}$.

Acknowledgement The authors wish to thank K en-iti Sato and M asaaki T suchiya for useful comments during the preparation of this article. We also wish to thank the anonymous refer ee who pointed out errors and many misprints. Especially, proofs of Theorem 4.3 and Lemma 4.3 became simpler and the statement of Theorem 6.3 has been extended following his/her advice. The ..rst author acknowledges the support of grants B F M 2000-807 and B FM 2000-0598. 


\section{R eferences}

[1] A smussen, S.(1998). Subexponential asymptotics for stochastic processes: extremal behaviour, stationary distributions and ..rst passage probabilities. Ann. A ppl. Probab. 8, 354-374.

[2] B rockwell, P. J ., Resnick, S. J ., T weedie, R. L.(1982). Storage processes with general release rule and additive inputs. Adv. A ppl. Probab. 14, 392-433.

[3] Chistyakov, V.P.(1964). A theorem on sums of independent positive random variables and its applications to branching processes. Theory P robability A ppl. 9, 640-648.

[4] Deshmukh, S., Pliska, S. (1980). O ptimal consumption and exploration of nonrenewable resources under uncertainty, E conometrica, 48, 177-200.

[5] Dharmadhicari, S., J oag-dev, K .( 1988). Unimodality, Convexity and Applications, A cademic Press, San Diego.

[6] Embrechts, P., Goldie, C. M., Veraverbeke, N., (1979). Subexponentiality and in..nite divisibility, Z. Wahrscheinlichkeitstheorie verw. Gebiette 49, 335-347.

[7] Feller, W ., (1971). A n Introduction to Probability theory and its A pplications. Vol 2-second edition, W iley, New York-L ondon-Sydney-Toronto.

[8] G rigoriu, M., Samorodnitsky, G. (2002). Tails of solutions of certain nonlinear stochastic dixerential equations driven by heavy tailed Lévy motions, M ini-proceedings: 2nd M aPhySto Conference on Lévy Processes: Theory and A pplications. MaP hySto, 202-205.

[9] J anicki, A., Weron, A . (1994). Simulation and Chaotic B ehavior of ${ }^{\circledR}$ stable stochastic Processes. Marcel Dekker, New Y ork, Basel, Hong Kong.

[10] K endall, D. G. (1957). Some problems in the theory of dams. J . R oy. Statist. Soc. Ser. B. 19, 207-212.

[11] Rosinski, J ., Samorodnitsky, G. (1993). Distributions of subadditive functionals of sample paths of in..nitely divisible processes. A nn. Probab. 21, 996-1014.

[12] Sato, K., (1999). Lévy processes and in..nitely divisible distributions. Cambridge University Press, Cambridge.

[13] Sato, K., Yamazato, M. (1984). Operator-selfdecomposable distributions as limit distributions of processes of Ornstein-Uhlenbeck type. Stochastic Processes and their A pplications 17, 73-100.

[14] Sigman, K., Yao, D. (1994). Finite moments for inventory processes. A nn. A ppl. Probab. 4, 765-778. 